\title{
Obstructive Sleep Apnea is Related with the Risk of Retinal Vein Occlusion
}

This article was published in the following Dove Press journal:

Nature and Science of Sleep

\author{
Wencui Wan ${ }^{1, *}$ \\ Zhen $\mathrm{Wu}^{2, *}$ \\ Jia $\mathrm{Lu}^{2, *}$ \\ Weiwei Wan' \\ Jing $\mathrm{Gao}^{3}$ \\ Hongxia $\mathrm{Su}^{4}$ \\ Wei Zhu (iD ${ }^{5}$
}

'Department of Ophthalmology, First Affiliated Hospital of Zhengzhou University, Zhengzhou, People's Republic of China; ${ }^{2}$ Department of Ear, Nose, and Throat, Changshu No. 2 People's Hospital, Changshu, People's Republic of China; ${ }^{3}$ Department of Ophthalmology, First Hospital Affiliated to Soochow University, Suzhou, Jiangsu Province, People's Republic of China; ${ }^{4}$ Department of Rhinology, First Affiliated Hospital of Zhengzhou University, Zhengzhou, People's Republic of China; ${ }^{5}$ Department of Ophthalmology, Changshu No. 2 People's Hospital, Changshu, People's Republic of China

*These authors contributed equally to this work
Background: Retinal vein occlusion (RVO) was a vision-threatening retinal vascular disorder, however, the relationship between obstructive sleep apnea (OSA) and RVO risk remained unclear.

Methods: A total of 45 RVO cases and 45 controls between April 2018 and April 2020 were included. All the participants underwent full-night polysomnography (PSG) and thus detected the severity of OSA. Besides, the relationship between the apnea-hypopnea index (AHI) and oxidative and inflammatory biomarkers, including 8-hydroxy-2 deoxyguanosine (8-OHdG), C-reactive protein (CRP), interleukin 1 beta (IL1 $\beta$ ), interleukin 6 (IL6) and tumor necrosis factor alpha $(\mathrm{TNF} \alpha)$ were detected. The incidences of macular edema (ME) and neovascular glaucoma (NVG) were detected in a three-months follow-up.

Results: In this case-control study, it was found that OSA incidence was increased in the RVO cases comparing with the cataract controls. Advanced analyses about the RVO subtypes demonstrated that incidence of OSA was higher in the central RVO (CRVO) cases comparing with branch RVO (BRVO) cases. Plasma samples from OSA cases demonstrated relatively higher concentrations of oxidative stress parameters and inflammatory biomarkers, including 8-OHdG, CRP, IL1 $\beta$, and IL6, in the RVO cases. Significant linear correlations between AHI and oxidative/inflammatory biomarkers were detected, and advanced analyses on the OSA subtypes demonstrated that these biomarkers were significantly higher in cases with later stages of OSA. In a three months follow-up, an impaired visual activity improvement rate and increased ME incidence in the OSA group among all the RVO cases were detected.

Conclusion: OSA was related with an increased incidence of RVO. Besides, OSA would lead to increased oxidative and inflammatory biomarkers concentrations in the RVO cases. OSA could be used as a harmful prognostic factor of visual activity improvement and ME incidences. These findings highlighted the role of OSA in the development of RVO.

Keywords: obstructive sleep apnea, retinal vein occlusion, risk factor, prognosis, oxidative stress

\section{Introduction}

Retinal vein occlusion (RVO) was the second prevalent retinal vascular disorder and dramatically affected the visual function as well as life quality of the patients. ${ }^{1-3}$ The long-term prognosis was influenced by RVO subtypes (branch or central RVO), therapeutic managements and complications incidence. The symptoms secondary to RVO, including macular edema (ME) and neovascular glaucoma (NVG), would cause severe vision loss and even blindness in some NVG cases. ${ }^{4}$ The risk factors of RVO were multifactorial and complex, and both genetic and environmental factors were related with RVO incidence. ${ }^{5}$ Besides, RVO risk was significantly increased with the appearance of systemic disorders, including
Department of Rhinology, First Affiliated

Hospital of Zhengzhou University,

Zhengzhou, People's Republic of China

Tel +86- I37726I 29234

Email hxs_ent@I63.com

Wei Zhu

Department of Ophthalmology, Changshu No. 2 People's Hospital, No. 99, Xiannan

Street, Changshu, Jiangsu, 215000 , People's

Republic of China

Tel +86- I37762I 2565

Email shzhuwei0722@I63.com
Nature and Science of Sleep 2021:13 273-28I 
hypertension, diabetes and stroke, as well as ocular diseases, including glaucoma. Considering the relatively poor prognoses and high costs of treatment for RVO, it was important to detect the RVO-causing factors and thus provide us more knowledge in the primary prevention of RVO. $^{6}$

RVO was a hypercoagulability related disorder and could be divided into branch RVO (BRVO) and central RVO (CRVO) according to the occluded location. The detailed pathological mechanisms of the development of RVO included functional damages of the vascular wall, increased thrombosis related blood contents, and hemodynamic changes. $^{7}$ It was also reported that chronic hypoxia would lead to a significant microvascular damage and thus induce thrombosis formation in retinal vein. ${ }^{8}$ Obstructive sleep apnea (OSA) had been reported to be a key illness causing systemic hypoxia and would affect the incidence and prognosis of RVO. $^{9}$

As a prevalent sleep disorder, the potential harms of OSA were usually ignored by the patients, however, OSA was a risk factor of different orders, including hypertension and stroke. ${ }^{10,11}$ In recent years, mounting evidences demonstrated that glaucoma incidence was increased in the OSA cases comparing with the controls. ${ }^{12}$ Based on the fact that hypertension, stroke and glaucoma were recognized as risk factors of RVO, it was reasonable to conjecture that OSA was related with RVO risk as well. ${ }^{13}$ The association between OSA and RVO was reported in recent cross-sectional and case series studies, and it was found that a higher rate of OSA was detected in the RVO cases. ${ }^{14}$ To further demonstrate the potential influence of OSA on the RVO incidence, this current case-control study aimed to (1) detect and compare OSA incidence rates in both RVO cases, and control subjects, (2) clarify the relationship of OSA and RVO severity and prognoses (eg, RVO subtype, vision damage and ME/NVG incidences) and (3) the effects OSA on the oxidative and inflammatory biomarkers, including 8-hydroxy-2 deoxyguanosine (8-OHdG), C-reactive protein (CRP), interleukin 1 beta (IL1 $\beta$ ), interleukin 6 (IL6) and tumor necrosis factor alpha $(\mathrm{TNF} \alpha)$, in RVO patients.

\section{Methods}

\section{Study Designs and Ethics Statements}

This case-control study included 45 RVO cases and 45 age- and sex-matched controls who came to Changshu No.2 Hospital between April 2018 and April 2020. This current study was complied with the Declaration of Helsinki and approved by the institutional Medical Ethics Committee of Changshu No.2 Hospital (2020-KY-001). Informed consents were obtained from both RVO cases and control subjects.

\section{Participants Inclusion and Exclusion}

The inclusion criteria of RVO group were RVO cases confirmed by fundus photography and fluorescein angiography. The control group included cataract patients ruling out any retinal pathologies. All the RVO cases should finish a three-months follow-up.

The exclusion criteria of both RVO cases and cataract controls were as the following: (1) any cases with cardiovascular disorders, stroke, diabetes, rheumatic immune system diseases, malignant tumors as well as sleep disorders, such as depression and insomnia; (2) patients with other fundus or vitreous disorders, including diabetic retinopathy and age-related macular degeneration; (3) past glaucoma, uveitis and neovascularization glaucoma history; (4) previous vitreoretinal surgery; (5) patients with allergies or general conditions that do not allow or do not cooperate with the examination or treatment; (6) recurrent RVO cases; and (7) the participants who cannot complete the 3-month follow-up study. The diagnoses of RVO was made according to Retinal Vein Occlusions Preferred Practice Pattern ${ }^{\circledR}$. 15

\section{OSA Assessment}

All the participants underwent full-night polysomnography (PSG) in the ear-nose-throat department of Changshu No.2 Hospital. Sleep during daytime, coffee, strong tea, or alcohol consumption, or sedative drugs intake were avoided. The PSG would be conducted with the Respironics Alice5 system in the department of Ear-Nose-Throat of Changshu No.2 Hospital. The sleep monitor assay was conducted from 21:00 on the night of admission to the morning of the next day and a total of more than 7 hours of sleep duration were analyzed. The analyses data included sleep apnea-hypopnea index (AHI), oxygen reduction index (ODI), minimum oxygen saturation $\left(\operatorname{minSPO}_{2}\right)$ and time of blood oxygen saturation below 90\% (CT90). An AHI < 5 would be considered negative for OSA and thus could be diagnosed as non-OSA. For OSA cases, the severity of OSA would be classified into mild, moderate, and severe OSA with an AHI value of 5 15, 16 30 and over 30 (number of events/hour), respectively. 


\section{Participants Management and Follow-Up}

After being included in this current study, the participants would finish a comprehensive ophthalmological examination. The best corrected visual activity (BCVA) would be conducted with international standard logarithmic visual acuity chart after subjective refraction (TOPCON IS-600, Japan) and retinoscopy (Heine beta 200, Germany). The intraocular pressure (IOP) was conducted using a noncontact tonometer (NIDEK, Japan) and the mean value of three independent detections would be used in the final analyses. The macular thickness would be conducted with optical coherence tomography (OCT, Zeiss cirrus HD-OCT 500, Germany). All the RVO cases would be divided into CRVO and BRVO groups and the disease duration would be recorded for each individual. A follow-up would be conducted three months after the administration. The BCVA improvement as well as incidences of ME and NVG would be recorded for advanced analyses. The occurrence of ME would be defined as the thickness of the foveal retina before treatment over $250 \mu \mathrm{m}$ or appearance of obvious cystic changes. The appearances of iris neovascularization in the RVO cases would be classified as the NVG cases.

\section{Laboratory Test}

The peripheral blood samples would be obtained from all the participants for the oxidative stress and inflammatory markers detection. After centrifuging $2 \mathrm{~mL}$ of anticoagulant whole blood for $15 \mathrm{~min}$ at room temperature, the plasma samples would be extracted and stored at $-80^{\circ} \mathrm{C}$ until use. The CRP concentration was detected using an immunofluorescence detector (FS-112, China). For the detection of IL-1 $\beta$, IL-6, TNF $\alpha$ and 8-OHdG in the plasma samples, commercial enzyme-linked immunosorbent assay (ELISA) kit (R\&D, USA) would be performed following according to the manufacturers' instructions.

\section{Statistical Analysis}

All the statistical analyses were conducted with GraphPad Prism 8.3 (GraphPad Software Inc, CA, USA). The continuous data, including oxidative stress and inflammatory markers, were present in mean \pm standard deviation (SD). Unpaired $t$-test and $\chi^{2}$ tests would be used to compare the incidence of OSA and non-OSA groups among all the participants. The correlation analyses between two independent parameters were conducted with Pearson correlation analyses. $P$ values of less than 0.05 were considered statistically significant.

\section{Results}

\section{Clinical Characteristics and Laboratory} Data of RVO Cases and Cataract Controls

The clinical characteristics and laboratory data of 45 controls and 45 RVO cases were presented in Table 1. Age and gender distributions were comparable between

Table I Clinical Characteristics and Laboratory Data of RVO Cases and Cataract Controls

\begin{tabular}{|c|c|c|c|}
\hline Subjects & $\begin{array}{c}\text { Control } \\
(n=45)\end{array}$ & $\begin{array}{c}\text { RVO } \\
(n=45)\end{array}$ & $P$ value \\
\hline \multicolumn{4}{|l|}{ General information } \\
\hline Gender (male, \%) & $25(55.6 \%)$ & 17 (37.8\%) & 0.139 \\
\hline Age (years) & $60.33 \pm 9.46$ & $60.07 \pm 10.33$ & 0.962 \\
\hline BMI $\left(\mathrm{Kg} / \mathrm{m}^{2}\right)$ & $24.80 \pm 6.04$ & $28.18 \pm 6.25$ & 0.025 \\
\hline Hypertension (n, \%) & $9(20 \%)$ & $21(46.7 \%)$ & 0.013 \\
\hline Hyperlipidemia (n, \%) & $19(42.2 \%)$ & 19 (42.2\%) & 0.584 \\
\hline BCVA logMAR & $0.23 \pm 0.11$ & $0.14 \pm 0.08$ & 0.001 \\
\hline IOP $(\mathrm{mmHg})$ & $15.53 \pm 2.97$ & $16.60 \pm 3.43$ & 0.122 \\
\hline \multicolumn{4}{|l|}{ OSA related parameters } \\
\hline OSA incidence & 17 (37.8\%) & 30 (66.7\%) & 0.011 \\
\hline \multicolumn{4}{|l|}{ OSA stage } \\
\hline Mild & 5 (29.4\%) & $5(16.7 \%)$ & \multirow[t]{3}{*}{0.559} \\
\hline Moderate & $6(35.3 \%)$ & $14(46.6 \%)$ & \\
\hline Severe & 6 (35.3\%) & II (36.7) & \\
\hline $\mathrm{AHI}$ & $12.06 \pm 16.34$ & $20.00 \pm 17.25$ & 0.039 \\
\hline СТ90 & $0.81 \pm 1.45$ & $1.65 \pm 2.23$ & 0.049 \\
\hline ODI & $4.20 \pm 5.38$ & $5.84 \pm 6.54$ & 0.325 \\
\hline $\operatorname{minSPO} \mathrm{O}_{2}$ & $89.47 \pm 9.06$ & $86.82 \pm 9.04$ & 0.263 \\
\hline \multicolumn{4}{|c|}{ Oxidative and inflammatory parameters } \\
\hline 8-OHdG (ng/mL) & $158.78 \pm 48.36$ & $242.29 \pm 68.50$ & $<0.001$ \\
\hline CRP $(\mathrm{mg} / \mathrm{l})$ & $7.30 \pm 2.74$ & $13.83 \pm 4.94$ & $<0.001$ \\
\hline $\mathrm{ILI} \beta(\mathrm{pg} / \mathrm{mL})$ & $193.25 \pm 50.87$ & $353.48 \pm|| 9.5 \mid$ & $<0.001$ \\
\hline IL6 (pg/mL) & $47.27 \pm 13.55$ & $84.83 \pm 49.86$ & $<0.001$ \\
\hline
\end{tabular}

Notes: Comparison among groups was calculated using unpaired $t$-test and chi-square exact test; the comparisons with a significant difference were marked in bold.

Abbreviations: RVO, retinal vein occlusion; BMI, body mass index; BCVA, best corrected visual activity; IOP, intraocular pressure; OSA, obstructive sleep apnea; $\mathrm{AHI}$, apnea hypopnea index; CT90, time of blood oxygen saturation below $90 \%$; ODI, oxygen reduction index, minSPO2, minimum oxygen saturation; 8-OHdG, 8-hydroxy-2 deoxyguanosine; CRP, C-reactive protein; IL-I $\beta$, interleukin-I $\beta$; IL-6, interleukin-6; TNF $\alpha$, tumor necrosis factor alpha. 
controls and cases, whereas body mass index (BMI), hypertension incidence, and best corrected visual activity (BCVA) $\log$ MAR were significantly impaired in the RVO group ( $P=0.025,0.013$ and 0.001 , respectively). No significant difference was detected in the hyperlipidemia incidence and IOP between RVO cases and controls $(P>0.05)$. When the OSA incidence and related parameters considered, a significant increased incidence rate was detected in the RVO group (37.8\% vs $66.7 \%$, $P=0.011)$. After comparing the detailed OSA related parameters, higher AHI and CT90 was detected in the RVO group $(12.06 \pm 16.34$ vs $20.00 \pm 17.25, P=0.039$ and $0.81 \pm 1.45$ vs $1.65 \pm 2.23, P=0.049$, respectively). While in the advanced analyses, it was found that there was no significant difference in OSA stages, ODI or minSPO2 between RVO cases and controls $(P>0.05)$. In the analyses of oxidative stress related parameters and inflammatory biomarkers in RVO and control group, a significant increased plasma 8-OHdG, CRP, IL1 $\beta$ and IL6 concentrations (158.78 \pm 48.36 vs 242.29 $\pm 68.50, P<0.001 ; 7.30 \pm 2.74$ vs $13.83 \pm 4.94, P<0.001$; $193.25 \pm 50.87$ vs $353.48 \pm 119.51, P<0.001 ; 47.27 \pm 13.55$ vs $84.83 \pm 49.86, P<0.001$, respectively) were detected in the RVO cases.

\section{Clinical Characteristics and Laboratory Data of CRVO and BRVO Cases}

In advanced analyses on the distribution of clinical characteristics, OSA related parameters as well as oxidative and inflammatory factors in CRVO and BRVO cases were analyzed. Comparing with the BRVO cases, a significant increased BMI was detected in the CRVO cases (25.31 \pm 5.09 vs $30.69 \pm 6.17, P=0.003)$. Neither significant distribution of gender, hypertension or hyperlipidemia, age, BCVA, nor IOP were detected in BRVO and CRVO cases $(P>0.05)$. The OSA incidence was higher in the CRVO cases comparing with the BRVO cases $(47.6 \%$ vs $83.3 \%, P=0.025)$, however, a reduced $\operatorname{minSPO}_{2}$ was found among all the OSA relatedparameters $(89.43 \pm 9.58$ vs $84.23 \pm 8.56, P=0.040$ ). After analyzing the oxidative and inflammatory factors in the RVO subtypes, it was found that plasma 8-OHdG and IL1 $\beta$ concentrations were higher in the CRVO cases $(217.10 \pm 47.87$ vs $264.32 \pm 76.80$, $P=0.019$ and $307.30 \pm 110.08$ vs $393.88 \pm 114.61, P=0.014$, respectively). All the detailed information was presented in Table 2.
Table 2 Clinical Characteristics and Laboratory Data of CRVO and BRVO Cases

\begin{tabular}{|c|c|c|c|}
\hline Subjects & $\begin{array}{l}\text { BRVO } \\
(n=21)\end{array}$ & $\begin{array}{l}\text { CRVO } \\
(n=24)\end{array}$ & $P$ value \\
\hline \multicolumn{4}{|l|}{ General information } \\
\hline Gender (male, \%) & 7 (33.3\%) & $10(41.7 \%)$ & 0.759 \\
\hline Age (years) & $58.38 \pm 10.77$ & $61.54 \pm 9.91$ & 0.311 \\
\hline BMI $\left(\mathrm{Kg} / \mathrm{m}^{2}\right)$ & $25.31 \pm 5.09$ & $30.69 \pm 6.17$ & 0.003 \\
\hline Hypertension (n, \%) & II (52.4\%) & $10(41.7 \%)$ & 0.556 \\
\hline Hyperlipidemia (n, \%) & 7 (33.3\%) & $12(50.0 \%)$ & 0.365 \\
\hline BCVA logMAR & $0.91 \pm 0.39$ & $1.06 \pm 0.51$ & 0.298 \\
\hline IOP $(\mathrm{mmHg})$ & $16.19 \pm 3.87$ & $16.96 \pm 3.03$ & 0.460 \\
\hline \multicolumn{4}{|l|}{ OSA related parameter } \\
\hline OSA incidence & $10(47.6 \%)$ & $20(83.3 \%)$ & 0.025 \\
\hline \multicolumn{4}{|l|}{ OSA stage } \\
\hline Mild & $2(9.5 \%)$ & $3(12.5 \%)$ & \multirow[t]{3}{*}{0.866} \\
\hline Moderate & $4(19.0 \%)$ & $10(41.7 \%)$ & \\
\hline Severe & $4(19.0 \%)$ & $7(29.2 \%)$ & \\
\hline $\mathrm{AHI}$ & $17.75 \pm 16.40$ & $21.97 \pm 18.06$ & 0.420 \\
\hline СТ90 & $1.39 \pm 2.13$ & $2.22 \pm 2.30$ & 0.187 \\
\hline ODI & $5.18 \pm 6.89$ & $7.10 \pm 6.93$ & 0.318 \\
\hline $\operatorname{minSPO}{ }_{2}$ & $89.43 \pm 9.58$ & $84.23 \pm 8.56$ & 0.040 \\
\hline \multicolumn{4}{|c|}{ Oxidative and inflammatory parameters } \\
\hline 8-OHdG (ng/mL) & $217.10 \pm 47.87$ & $264.32 \pm 76.80$ & 0.019 \\
\hline CRP $(\mathrm{mg} / \mathrm{l})$ & $13.30 \pm 6.06$ & $14.30 \pm 3.78$ & 0.505 \\
\hline $\mathrm{ILI} \beta(\mathrm{pg} / \mathrm{mL})$ & $307.30 \pm 110.08$ & $393.88 \pm|| 4.6 \mid$ & 0.014 \\
\hline IL6 (pg/mL) & $71.10 \pm 32.34$ & $96.84 \pm 59.36$ & 0.084 \\
\hline
\end{tabular}

Notes: Comparison among groups was calculated using unpaired $t$-test and chisquare exact test; the comparisons with a significant difference were marked in bold.

Abbreviations: BRVO, branch retinal vein occlusion; CRVO, central retinal vein occlusion; BMI, body mass index; BCVA, best corrected visual activity; IOP, intraocular pressure; OSA, obstructive sleep apnea; AHI, apnea hypopnea index; CT90, time of blood oxygen saturation below $90 \%$; ODI, oxygen reduction index; minSPO2, minimum oxygen saturation; 8-OHdG, 8-hydroxy-2 deoxyguanosine; CRP, C-reactive protein; IL-I $\beta$, interleukin-I $\beta$; IL-6, interleukin-6; TNF $\alpha$, tumor necrosis factor alpha.

\section{Correlations Between Clinical/ Laboratory Characteristics and $\mathrm{AHI}$}

As AHI was one of the most important parameters of OSA, the correlations between clinical/laboratory characteristics and AHI value were analyzed in both all the 
Table 3 Correlations Between Clinical Information and AHI

\begin{tabular}{|l|c|c|c|c|}
\hline \multirow{2}{*}{ Subjects } & \multicolumn{2}{|c|}{ All Participants } & \multicolumn{2}{c|}{ RVO Cases } \\
\cline { 2 - 5 } & $\boldsymbol{r}$ & $\boldsymbol{P}$ & $\boldsymbol{r}$ & $\boldsymbol{P}$ \\
\hline Age & 0.037 & 0.730 & 0.050 & 0.742 \\
BMI & 0.273 & $\mathbf{0 . 0 0 9}$ & 0.463 & $\mathbf{0 . 0 0 I}$ \\
BCVA logMAR & 0.183 & 0.084 & 0.153 & 0.315 \\
IOP (mmHg) & 0.006 & 0.958 & 0.035 & 0.818 \\
8-OHdG (ng/mL) & 0.269 & $\mathbf{0 . 0 1 0}$ & 0.419 & 0.004 \\
CRP (mg/l) & 0.309 & $\mathbf{0 . 0 0 3}$ & 0.212 & 0.161 \\
ILI $\beta(\mathrm{pg} / \mathrm{mL})$ & 0.319 & $\mathbf{0 . 0 0 2}$ & 0.216 & 0.154 \\
IL6 (pg/mL) & 0.470 & $<0.001$ & 0.516 & $<0.001$ \\
\hline
\end{tabular}

Note: The linear correlations with a significant difference were marked in bold. Abbreviations: RVO, retinal vein occlusion; BMI, body mass index; BCVA, best corrected visual activity; IOP, intraocular pressure; 8-OHdG, 8-hydroxy-2 deoxyguanosine; CRP, C-reactive protein; IL-I $\beta$, interleukin-I $\beta$; IL-6, interleukin-6.

participants and RVO cases. As shown in Table 3, a significant linear correlation between BMI, 8-OHdG, CRP, IL1 $\beta$ and IL6 concentrations with $r$ values of $0.273, \quad 0.269,0.309, \quad 0.319$ and 0.470 , respectively ( $P=0.009,0.010,0.003,0.002$ and $<0.001$, respectively). Advanced analyses on the correlations of clinical and experimental characteristics and the AHI value demonstrated that increased BMI and IL6 concentrations were related with a higher AHI $(r=0.463, P=0.001$, and $r=0.516$, $P<0.001$, respectively).

\section{Oxidative and Inflammatory Biomarkers in the Non-OSA and OSA Cases}

As both oxidative and inflammatory biomarkers were correlated with the OSA parameter, AHI, the plasma 8-OHdG, CRP, IL1 $\beta$ and IL6 concentrations in non-OSA, mild, moderate and severe OSA groups were detected. Significant differences in the $8-\mathrm{OHdG}, \mathrm{CRP}, \mathrm{IL} 1 \beta$ and IL6 contents were detected among different OSA stages, thus it indicated that OSA stages were related with the concentrations of circulating oxidative and inflammatory biomarkers. In advanced multiple comparisons, it was found that no significant difference was detected in the plasma 8-OHdG concentration in OSA of any stage comparing with the non-OSA cases $(P>0.05$, Figure 1A). When the CRP level was considered, a significant increased CRP level was detected in the severe OSA group comparing with the non-OSA group $(P=0.011$, Figure 1B). Two potent inflammatory factors, IL1 $\beta$ and IL6, were also detected in different OSA stages. Comparing with the non-OSA cases, higher concentration of IL1 $\beta$ and IL6 were detected in moderate/severe and severe OSA groups, respectively $(P=0.006$ and 0.026 , Figure $1 \mathrm{C}$ and $P<0.001$, Figure $1 \mathrm{D}$, respectively).

\section{The Short-Term Prognoses in RVO Cases with or without OSA}

As the RVO related complications significantly affected the visual quality of patients, the visual activity improvement, incidence of ME and neovascular glaucoma in RVO cases with or without OSA after three months follow-up were analyzed in this current study. The effective visual improvement was defined as more than two lines of visual activity improvement. As demonstrated in Table 4, a significantly decreased visual activity improvement was detected in the OSA group comparing with the non-OSA group (40.0\% vs $73.3 \%, P=0.036)$. Besides, a higher $\mathrm{ME}$ incidence rate was detected in the OSA group and it was almost two times higher risk compared with the RVO cases without OSA $(36.7 \%$ vs $13.3 \%, P=0.098)$. When the NVG was considered, one case of NVG was detected in the RVO patients with OSA, however, no significant difference was detected because of the low incidence rate (3.3\% vs $0.0 \%, P=0.669)$.

\section{Discussion}

In this case-control study, it was demonstrated that the OSA incidence was increased in the RVO group comparing with the cataract control group. Advanced analyses considering the RVO subtypes demonstrated that higher OSA risk was detected in the CRVO group comparing with BRVO group. Plasma from subjects with OSA demonstrated relatively high concentrations of oxidative stress and inflammatory biomarkers, including 8-OHdG, CRP, IL1 $\beta$ and IL6, in both all the participants and RVO cases. Significant linear correlations between these oxidative and inflammatory biomarkers and OSA parameter AHI were detected, and advanced analyses on the OSA subtypes showed that these biomarkers were significantly higher in cases with severer stages of OSA. In the three months follow-up, a decreased visual activity improvement rate and increased $\mathrm{ME}$ incidence in the OSA group among all the RVO cases were detected.

OSA, as an extremely common sleep disorder, had been reported to be related with various chronic diseases. In recent years, interesting findings between the association of OSA and glaucoma had attracted the attentions of ophthalmologists and sleeping disorder experts. ${ }^{12,13,16,17}$ After this, mounting evidence on the relationships between 

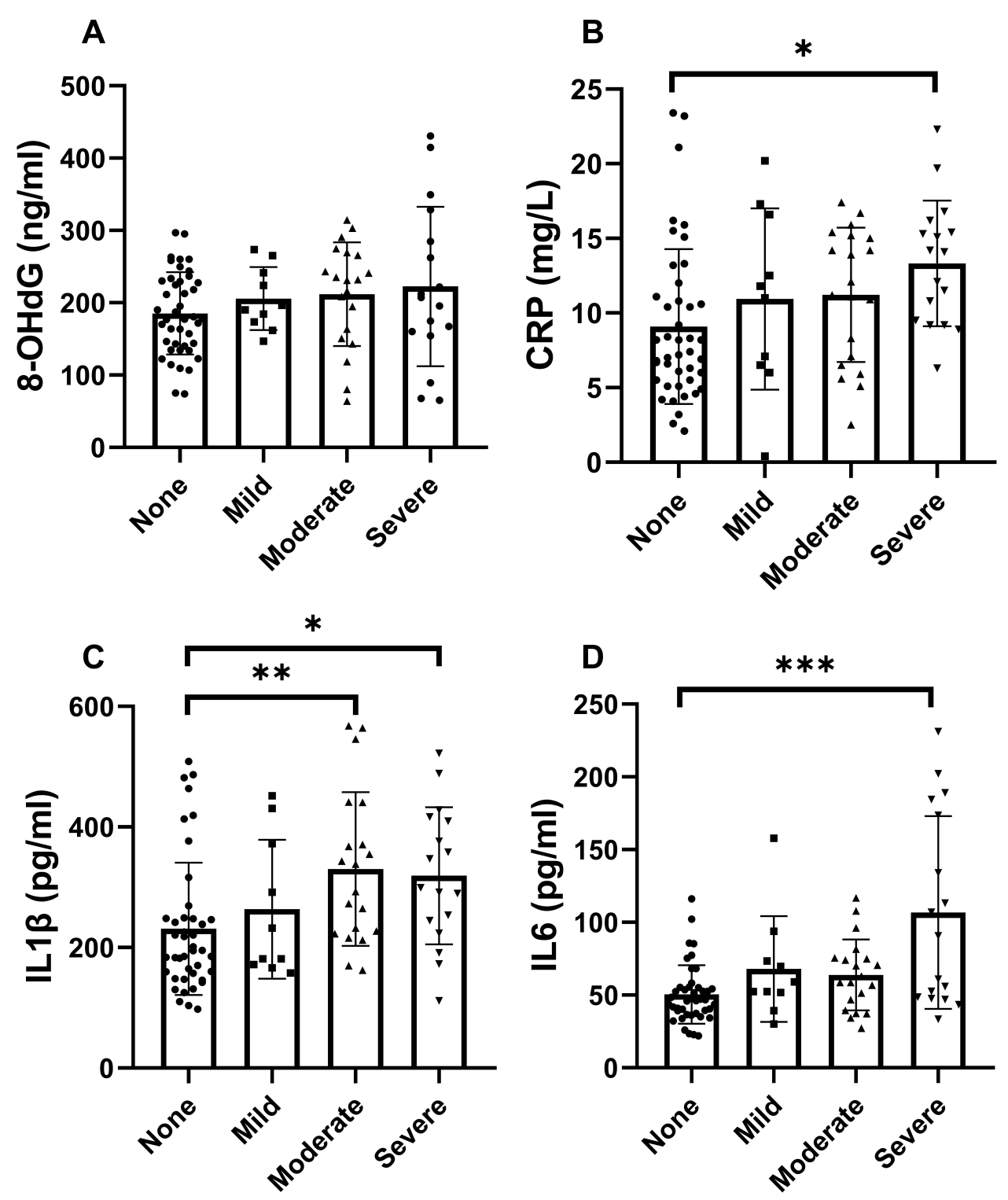

Figure I Oxidative and inflammatory biomarkers in the non-OSA and OSA cases in different stages. In advanced multiple comparisons, the plasma 8-OHdG concentration (A), CRP (B), ILI $\beta$ (C) and IL6 (D), in OSA of any stage comparing with the non-OSA cases. $* P<0.05, * * P<0.01$ and $* * * P<0.00$ I.

OSA and ocular disorders were reported and the potential mechanisms were studied as well. RVO was a thrombus formation-related disease and caused by the occlusion of retinal veins. As reported in previous studies, OSA had been reported to be related thrombosis, including myocardial infarction and stroke, ${ }^{18,19}$ thus it was reasonable to conjecture that OSA demonstrated a potential to affect the development of RVO to a certain extent. In a recent decade, it was found that OSA had been regarded as a risk factor of RVO based on population-based and crosssectional studies. In a case series with 114 RVO patients, ${ }^{20}$ the incidence of OSA in both BRVO and CRVO were analyzed and thus the results of this study confirmed that OSA could be regarded as a risk factor of RVO. In this current study based on both RVO cases and age- and gender-matched controls, it was found that OSA was significantly associated an increased risk of RVO. In advanced analyses stratified study based on the RVO subtypes, it was found that OSA was more common in the CRVO cases among all the participants with RVO. In consistent with previous observational studies, it was found that OSA could be regarded as a risk factor of RVO. More detailed mechanisms and potential benefits of OSA on RVO progression remained unclear.

OSA was a disorder causing systemic hypoxia and then leaded to a series of disorders substantially. In a previous 
Table 4 The Visual Activity Improvement and Incidence of Macular Edema and Neovascular Glaucoma in RVO Cases with or without OSA After Three Months Follow-Up

\begin{tabular}{|l|c|c|c|}
\hline Subjects & $\begin{array}{c}\text { Non-OSA } \\
(\mathbf{n}=15)\end{array}$ & $\begin{array}{c}\text { OSA } \\
(\mathbf{n}=\mathbf{3 0})\end{array}$ & P value \\
\hline $\begin{array}{l}\text { VA improvement } \\
\text { over 2 lines }\end{array}$ & II (73.3\%) & $12(40.0 \%)$ & $\mathbf{0 . 0 3 6}$ \\
\hline ME & $2(13.3 \%)$ & II (36.7\%) & $\mathbf{0 . 0 9 8}$ \\
\hline NVG & $0(0.0 \%)$ & I (3.3\%) & 0.669 \\
\hline
\end{tabular}

Notes: Comparison among groups was calculated using unpaired $t$-test and chisquare exact test; the comparisons with a significant difference were marked in bold.

Abbreviations: OSA, obstructive sleep apnea; VA, visual activity; ME, macular edema; NVG, neovascular glaucoma.

review by Marielle Mentek and colleagues, ${ }^{13}$ it summarized the effects of OSA on the incidences of the retinal disorders and optic nerve related disease. In this comprehensive review, the authors agreed that it is legitimate to question the role of OSA in the onset of RVO and highlighted the importance in analyzing the potential benefits of OSA management in the therapy of RVO. In the detailed pathophysiological mechanism, OSA would lead to a significant hypercoagulability state in response to systemic inflammation and oxidative stress. Clinical studies on the correlation between OSA and inflammation demonstrated that inflammation was the link between OSA and neural system degeneration. ${ }^{21-24}$ As abnormal inflammation was detected in the RVO cases, ${ }^{2,25}$ the management of OSA would help in the inflammatory status and thus provide potential benefits in the RVO therapy. Besides, anatomical structures showed that central artery and central vein located in the same adventitial sheath, and hypoxia and hypercapnia caused by severe OSA would lead to vasodilation of the retinal central artery thus compressing the retinal vein. This anatomy related explanation was reasonable in theory and confirmed by the fact that OSA was an independent risk factor of glaucoma. Oxidative stress and chronic inflammation had been reported to be risk factors of $\mathrm{RVO},{ }^{26,27}$ however, no previous studies demonstrated the effects of OSA on oxidative stress and inflammatory biomarkers in the RVO cases by now. As significantly up-regulated oxidative and inflammatory markers could be detected in the OSA cases, detection of the relationship between their expressions and OSA/RVO incidences would provide us important knowledge about the potential pathophysiological functions. In this current study, oxidative and inflammatory biomarkers were found to be related with both RVO risk and OSA incidence. Considering the significant linear correlation between these biomarkers and OSA index, up-regulated oxidative stress and inflammatory biomarkers could explain the effects of OSA in the development of RVO, at least in part.

In a more important point, we tried to detect the potential functions of OSA in the prognoses of RVO, including the visual activity improvement and ME/NVG incidence. The visual improvement was related with the life quality of RVO patients, while the incidence of ME/NVG would lead to following repeated anti-VEGF treatment and potential vitrectomy. ${ }^{2,28}$ In this study, it was found that visual activity improvement rate was lower in the RVO cases with OSA and it demonstrated that OSA might lead to a poor recovery of both anatomy and function of retina in RVO cases. ME was a key reason of vision loss in the RVO cases, while it was found that RVO was related a higher risk of ME incidence. This had never been reported in previous studies, and it provided us huge imagination in the potential benefits of OSA treatment in the prognosis of RVO cases. When the NVG incidence was considered, only one case was detected in the OSA group and this might because of the relative short-term follow-up and effective management of RVO cases. However, the short-term follow-up could not reflect the long-term outcome of the RVO cases, we proposed a retrospective cohort study with a relative huge number of participants to examine the effects of OSA on the prognosis of RVO. Continuous positive airway pressure (CPAP) treatments were applied in the management of OSA, and CPAP treatment would alleviate the inflammatory status in OSA patients. ${ }^{29}$ The influence of CPAP on the therapy response as well as long-term prognosis would be conducted in advanced well-designed clinical trials.

There were several strengths in this current study. First, the examination of oxidative and inflammatory biomarkers helped us to deepen the understanding of potential effects of OSA on RVO incidence. Second, all the OSA diagnoses were made by PSG, ${ }^{30}$ which was the gold standard of OSA diagnoses. This accurate diagnostic method provided a conclusive basis of this case-control study. However, there were several limitations that could not be ignored. First, even PSG could be easily operated, it was still time and manpower consuming operation thus it limited the included participants in this study. Second, the cataracts without any retinal disorders were adopted as controls in this study, and it might be a potential confounding factor 
in this study. Even cataract patients were usually used as controls in the retinal disorders and the retinal status should be confirmed after cataract surgery in this age range, the potential effects of adopting cataracts cases in the control group should be confirmed in the following studies with huger number of participants.

In summary, we demonstrated that the OSA was related with an increased incidence of RVO, and advanced stratified analyses demonstrated that OSA was more common in CRVO cases. Besides, OSA would lead to increased concentrations of oxidative and inflammatory biomarkers and this might explain the pathological mechanism, at least in part. OSA would also be a potential prognostic factor of the visual activity improvement and $\mathrm{ME}$ incidences. These findings suggested that OSA contributed to the development and progression of RVO through up-regulation of oxidative stress and inflammatory progresses.

\section{Acknowledgments}

This work was supported in whole or in part by National Nature Science Foundation Project for Young Scientists of China (Grant No. 81700804), the Foundation for Young Medical Talents of Jiangsu Province (Grant No. QNRC2016211), Scientific Research Project of Jiangsu Health Commission (Z2019044) and Youth Project of Henan Provincial Health and Health Commission, Ministry of Education (SB201902008).

\section{Author Contributions}

All authors contributed to data analysis, drafting or revising the article, have agreed on the journal to which the article will be submitted, gave final approval of the version to be published, and agree to be accountable for all aspects of the work.

\section{Disclosure}

The authors declare no competing financial interests.

\section{References}

1. Khayat M, Williams M, Lois N. Ischemic retinal vein occlusion: characterizing the more severe spectrum of retinal vein occlusion. Surv Ophthalmol. 2018;63(6):816-850.

2. Noma H, Yasuda K, Shimura M. Cytokines and pathogenesis of central retinal vein occlusion. J Clin Med. 2020;9:11. doi:10.3390/ jcm9113457

3. Shalchi Z, Mahroo O, Bunce C, Mitry D. Anti-vascular endothelial growth factor for macular oedema secondary to branch retinal vein occlusion. Cochrane Database Syst Rev. 2020;7:CD009510. doi:10.10 02/14651858.CD009510.pub3
4. Casselholm de Salles M, Lindberg C, Epstein D. Neovascular glaucoma in patients with central retinal vein occlusion: a real-life study in the anti-VEGF era. Acta Ophthalmol. 2020;99(1):e7-e12. doi:10.1111/aos. 14500

5. Zhu W, Wu Y, Xu M, et al. Antiphospholipid antibody and risk of retinal vein occlusion: a systematic review and meta-analysis. PLoS One. 2014;10(4):e0122814. doi:10.1371/journal.pone.0122814

6. Hollo G, Aung T, Cantor LB, Aihara M. Cystoid macular edema related to cataract surgery and topical prostaglandin analogs: mechanism, diagnosis, and management. Surv Ophthalmol. 2020;65 (5):496-512. doi:10.1016/j.survophthal.2020.02.004

7. Xia JP, Wang S, Zhang JS. The anti-inflammatory and anti-oxidative effects of conbercept in treatment of macular edema secondary to retinal vein occlusion. Biochem Biophys Res Commun. 2019;508 (4):1264-1270. doi:10.1016/j.bbrc.2018.12.049

8. Ugurlu E, Pekel G, Altinisik G, Bozkurt K, Can I, Evyapan F. New aspect for systemic effects of COPD: eye findings. Clin Respir J. 2018;12(1):247-252. doi:10.1111/crj.12523

9. Eng VA, Leng T. Subthreshold laser therapy for macular oedema from branch retinal vein occlusion: focused review. Br J Ophthalmol. 2020;104(9):1184-1189. doi:10.1136/bjophthalmol-2019-315192

10. Hurvitz MS, Lesser DJ, Dever G, Celso J, Bhattacharjee R. Findings of routine nocturnal polysomnography in children with Down syndrome: a retrospective cohort study. Sleep Med. 2020;76:58-64. doi:10.1016/j.sleep.2020.10.003

11. Bahr K, Bopp M, Kewader W, et al. Obstructive sleep apnea as a risk factor for primary open angle glaucoma and ocular hypertension in a monocentric pilot study. Respir Res. 2020;21(1):258. doi:10.1186/ s12931-020-01533-7

12. Shah SM, Bakri SJ. Obstructive sleep apnea evaluation in retinal vein occlusion patients: an opportunity for multidisciplinary care? Can J Ophthalmol. 2020;55(4):284-285. doi:10.1016/j.jcjo.2020.07.002

13. Mentek M, Aptel F, Godin-Ribuot D, Tamisier R, Pepin JL, Chiquet C. Diseases of the retina and the optic nerve associated with obstructive sleep apnea. Sleep Med Rev. 2018;38:113-130. doi:10.1016/j.smrv.2017.05.003

14. Kwon HJ, Kang EC, Lee J, Han J, Song WK. Obstructive sleep apnea in patients with branch retinal vein occlusion: a preliminary study. Korean J Ophthalmol. 2016;30(2):121-126. doi:10.3341/kjo.2016. 30.2.121

15. Pulido JS, Flaxel CJ, Adelman RA, Hyman L, Folk JC, Olsen TW. Retinal vein occlusions preferred practice pattern $(\mathrm{R})$ ) guidelines. Ophthalmology. 2016;123(1):P182-208. doi:10.1016/j.ophtha.2015. 10.045

16. Wong B, Fraser CL. Obstructive sleep apnea in neuro-ophthalmology. $J$ Neuroophthalmol. 2019;39(3):370-379. doi:10.1097/WNO.0000 000000000728

17. Santos M, Hofmann RJ. Ocular manifestations of obstructive sleep apnea. J Clin Sleep Med. 2017;13(11):1345-1348. doi:10.5664/jcsm. 6812

18. Yamamoto S, Yamaga T, Nishie K, Nagata C, Mori R. Positive airway pressure therapy for the treatment of central sleep apnoea associated with heart failure. Cochrane Database Syst Rev. 2019;12:CD012803. doi:10.1002/14651858.CD012803.pub2

19. Geissenberger F, Schwarz F, Probst M, et al. Obstructive sleep apnea is associated with pulmonary artery thrombus load, disease severity, and survival in acute pulmonary embolism. Clin Res Cardiol. 2020;109(1):13-21. doi:10.1007/s00392-019-01479-x

20. Agard E, El Chehab H, Vie AL, Voirin N, Coste O, Dot C. Retinal vein occlusion and obstructive sleep apnea: a series of 114 patients. Acta Ophthalmol. 2018;96(8):e919-e925. doi:10.1111/aos.13798

21. Orru G, Storari M, Scano A, Piras V, Taibi R, Viscuso D. Obstructive sleep apnea, oxidative stress, inflammation and endothelial dysfunction-an overview of predictive laboratory biomarkers. Eur Rev Med Pharmacol Sci. 2020;24(12):6939-6948. doi:10.26355/ eurrev_202006_21685 
22. Rha MS, Kim CH, Yoon JH, Cho HJ. Association between the neutrophil-to-lymphocyte ratio and obstructive sleep apnea: a meta-analysis. Sci Rep. 2020;10(1):10862. doi:10.1038/s41598-02067708-w

23. Fernandez-Bello I, Monzon Manzano E, Garcia Rio F, et al. Procoagulant state of sleep apnea depends on systemic inflammation and endothelial damage. Arch Bronconeumol. 2020. doi:10.1016/j. arbres.2020.11.017

24. Weihs A, Frenzel S, Wittfeld K, et al. Associations between sleep apnoea and advanced brain ageing in a large-scale population study. Sleep. 2020. doi:10.1093/sleep/zsaa204

25. Hu Y, Yu Y, Bu Z, et al. Increased systemic heparanase in retinal vein occlusion is associated with activation of inflammation and thrombophilia. Retina. 2020;40(2):345-349. doi:10.1097/IAE.00000 00000002374

26. Chan TC, Wilkinson Berka JL, Deliyanti D, et al. The role of reactive oxygen species in the pathogenesis and treatment of retinal diseases. Exp Eye Res. 2020;201:108255. doi:10.1016/j.exer.2020.108255
27. Masuda T, Shimazawa M, Hara H. Retinal diseases associated with oxidative stress and the effects of a free radical scavenger (Edaravone). Oxid Med Cell Longev. 2017;2017:9208489. doi:10.115 $5 / 2017 / 9208489$

28. Liu H, Ma Y, Xu HC, Huang LY, Zhai LY, Zhang XR. Updates on the management of ocular vasculopathies with VEGF inhibitor conbercept. Curr Eye Res. 2020;1-10. doi:10.1080/02713683.20 20.1817490

29. Liu X, Ma Y, Ouyang R, et al. The relationship between inflammation and neurocognitive dysfunction in obstructive sleep apnea syndrome. $J$ Neuroinflammation. 2020;17(1):229. doi:10.1186/s12974-020-019 $05-2$

30. Barroso-Garcia V, Gutierrez-Tobal GC, Kheirandish-Gozal L, et al. Usefulness of recurrence plots from airflow recordings to aid in paediatric sleep apnoea diagnosis. Comput Methods Programs Biomed. 2020;183:105083. doi:10.1016/j.cmpb.2019.105083

\section{Publish your work in this journal}

Nature and Science of Sleep is an international, peer-reviewed, open access journal covering all aspects of sleep science and sleep medicine, including the neurophysiology and functions of sleep, the genetics of sleep, sleep and society, biological rhythms, dreaming, sleep disorders and therapy, and strategies to optimize healthy sleep.
The manuscript management system is completely online and includes a very quick and fair peer-review system, which is all easy to use. Visit http://www.dovepress.com/testimonials.php to read real quotes from published authors. 